\title{
空间柔性天线展开臂的半主动振动控制研究
}

\author{
王晓宇 ${ }^{1}$ 王浩威 $^{1}$ 间雪梅 $^{1}$ 从 强 ${ }^{1}$ 季宏丽 $^{2}$ 装进浩 $^{2}$ \\ (1. 北京空间飞行器总体设计部 北京 100094; \\ 2. 南京航空航天大学机械结构力学与控制国家重点实验室 南京 210016)
}

\begin{abstract}
摘要: 大型柔性天线展开臂结构由于其尺寸大, 阻尼和刚度小, 其振动呈现明显的低频特性, 且很难在短时间内迅速衰减, 从而影响天线工作性能。传统的被动控制大都采用阻尼材料, 通过增加阻尼的方法来进行控制, 这种控制方法对低频柔性结 构的控制效果是有限的。因此研究带负载的复合材料柔性展开臂结构的振动抑制问题, 提出用压电元件粘贴在展开臂结构表 面作为传感器和驱动器, 用基于同步开关阻尼技术的自适应半主动振动控制方法控制压电驱动器, 从而对展开臂结构实现振 动控制。完成了展开臂模型的建立和仿真分析, 建立了展开臂结构的开关阻尼半主动控制试验平台, 设计并制作了控制电路, 针对需要控制的结构一阶弯曲模态进行压电元件的合理布设。对不同激励下的振动进行了展开臂结构的控制试验, 试验结果 显示该方法对带负载柔性天线展开臂结构的振动具有良好的抑制效果, 对于初始位移为 $100 \mathrm{~mm}$ 的瞬态激励振动工况, 受控 系统等效阻尼比由 $0.6 \%$ 增加至 $4.03 \%$; 对于稳态振幅为 $50 \mathrm{~mm}$ 的稳态振动工况, 系统控制后结构端部振动幅值降低至 $10 \mathrm{~mm}$, 稳态振动幅值降低了 $16.843 \mathrm{~dB}$ 。在大型柔性结构中具有非常好的应用前景。
\end{abstract}

关键词: 同步开关阻尼技术; 自适应半主动振动控制; 柔性展开臂结构; 振动抑制

中图分类号: TG156

\section{Semi-active Vibration Suppression of Space Flexible Antenna Arm}

\section{WANG Xiaoyu $^{1} \quad$ WANG Haowei $^{1} \quad$ YAN Xuemei $^{1}$ CONG Qiang ${ }^{1} \quad$ JI Hongli $^{2}$ QIU Jinhao ${ }^{2}$}

(1. Beijing Institute of Spacecraft System Engineering, Beijing 100094;

State Key Laboratory of Mechanics and Control of Mechanical Structures, Nanjing University of Aeronautics and

Astronautics, Nanjing 210016)

\begin{abstract}
Large flexible antenna arm appears obviously low frequency vibration characteristics, for its light damp and feeble rigidity, caused by large scale. Meanwhile, this vibration cannot decay fast in a short time. Traditional passive control methods are mainly using the damping material, to implement the control by increasing damping, and the control effect of vibration problems on low-frequency flexible structures is limited. The vibration suppression problem on the flexible antennas arm structure of composite material with load is researched. Piezoelectric elements are used as the transducer and actuator, respectively, by sticking on the surface of the antennas arm structure. Vibration control on antennas arm is achieved, by using piezoelectric actuator drove by adaptive semi-active control method, based on synchronous switch damping technique. Simulation and analysis on flexible antenna arm is achieved. A test platform of switch damping semi-active control on antennas arm is built, on which the control circuit is designed and manufactured. In view of the need to control the first-order bending mode of the structure, a reasonable layout of the piezoelectric elements is set up. With the vibration control test under different excitation, the results show that the method has good inhibition to the antenna arm structure with load. For the case of transient excitation of a $100 \mathrm{~mm}$ initial displacement, the system equivalent damping ratio rises from $0.6 \%$ to $4.03 \%$. For the case of steady state excitation with a $50 \mathrm{~mm}$ amplitude, the displacement at the end of the structures after control is reduced to $10 \mathrm{~mm}$. The amplitude of steady state vibration decreases $16.843 \mathrm{~dB}$. It has immense application prospect in large flexible structure.
\end{abstract}

Key words: synchronous switch damping technique; adaptive semi-active control method; flexible antennas arm structure; vibration suppression

\section{0 前言}

随着航天技术的发展, 大型化是航天器结构的 一个重要发展趋势。大型柔性航天器结构具有尺寸

20190321 收到初稿, 20190617 收到修改稿
跨度大，固有频率低等特点。在轨工作时遭受各种 外界和内部因素的干扰, 激起低频、非线性、大幅 度的振动, 这种振动严重影响航天器的工作性能。 因此, 如何有效抑制大型柔性航天器结构的振动对 航天器的发展至关重要。其中基于智能结构的振动 控制方法是未来发展的一个重要方向。 
压电材料作为一种智能材料, 以其良好的机电 耦合特性, 在结构振动控制领域具有广泛的应用。 当前的压电振动控制系统中, 主要有主动、被动和 半主动三种。其中半主动控制不是将能量直接施加 在压电驱动器上, 而是将能量用于半主动控制回路 中, 通过控制串联在压电元件两端回路中的参数, 改变系统特性, 从而达到振动控制目的。其能量消 耗介于主动和被动之间, 具有良好的应用前景。

基于同步开关阻尼技术(Synochronited switch damping, SSD)的半主动振动控制方法, 具有控制 系统简单, 控制所需要的外界能量很小, 不需要精 确的结构振动模型, 且控制效果比较稳定, 适合于 宽频带的振动控制等优点。自法国 GUYOMAR 等 提出后 ${ }^{[1-4]}$, 得到了广大学者的特别关注, 有了长足 的发展。SSD 的形式有很多种, 最先提出来的是基于 短路同步开关阻尼技术(Short-circuit SDS, SSDS) ${ }^{[2]}$, 为了进一步提高控制效果, RICHARD 等于 2000 年 提出了基于电感同步开关阻尼技术(SDS inductance, $\mathrm{SSDI}$ )技术 ${ }^{[4]}$, 即在回路中串联一个电感 $\mathrm{L}$, 与压电 元件电容构成 LC 共振电路, 从而增大压电元件上 的控制电压, 提高了控制效果。为了进一步增大压 电元件上的电压, LEFEUVRE 于 2006 年提出了基 于外加电压源的同步开关阻尼技术(Synchronous switch damping voltage, SSDV) ${ }^{[5]}$, 即在 SSDI 的回 路中串联一个额外的电压源, 由于外加电压源的作 用, 使得电压进一步提高, 增加了振动控制效果。

GRASSO 于 2013 年提出了一种在低驱动电压和高 驱动电压下自感知驱动技术, 利用滞后模型来描述 这种自感知重构的非线性压电电容 ${ }^{[6]}$ 。2017 年, BAO 等 ${ }^{[7]}$ 采用自感知的 SSDV 方法实现了结构的半主动 振动控制, 并进行了试验验证。

在大柔性结构的振动控制研究中, 黄文虎等 ${ }^{[8]}$ 指 出压电元件等智能结构特别适合于大型柔性结构振 动控制的需要。日本的 MAKIHARA 等 ${ }^{[9-12]}$ 利用压电 叠堆对桁架结构进行了半主动振动控制研究。根据桁 架结构的振动特性和控制要求, 在桁架结构某些振动 位移较大关节处的内部粘贴了多个压电叠堆, 利用基 于同步开关阻尼技术的半主动方法成功的实现了多 模态振动控制, 获得了良好的控制效果。意大利学者 SABATINI 提出一种基于压电器件的优化自适应振动 控制, 并研究过程中, SABATINI 对压电元件的数量 和布置位置进行了分析与优化 ${ }^{[14]}$ 。2014 年, GARRIDO 等 ${ }^{[15]}$ 提出了由可变摩擦阻尼器组成, 通过 电缆与结构相连的新型半主动控制系统, 通过数值验 证了该方法在多自由结构中的应用, 说明了该方法比 被动控制更有效, 对预张力损失不敏感。
本文首次将自适应半主动振动控制技术应用到 大型天线展开臂结构中。建立了含有三个复合材料杆 件和两个弹簧等效关节的展开臂复杂模型的仿真模 型, 并搭建了在根部粘贴有限压电元件的天线展开臂 结构试验平台。通过多组工况的仿真分析和试验对 比, 在工程上验证了半主动振动控制技术对大型空间 结构由于外扰动引起的振动进行控制的有效性。

\section{1 自适应 SSDV 控制方法}

\section{1 半主动控制原理}

当结构在某阶共振频率附近发生振动时, 其机 电模型可以用单自由度的弹簧质量阻尼系统进行描 述, 机电模型如图 1 所示。

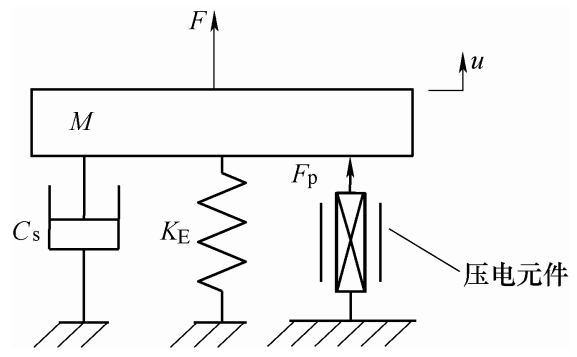

图 1 机电模型的示意图

假设包括压电元件在内的所有结构均为线弹 性, 由牛顿定律得如下微分方程

$$
M \ddot{u}+C_{s} \dot{u}+K_{E} u=F+F_{p}
$$

式中, $M$ 为结构等效模态质量; $C_{s}$ 为机械损失因子; $K_{E}$ 为当压电元件短路时结构的等效结构刚度; $u$ 为 模态质量位移; $F_{P}$ 为压电元件通过逆压电效应产生 的作用在机械结构上的力; $F$ 为外部激振力。

如果被控结构上埋入或粘贴压电元件时, 其机 电耦合方程为

$$
\left\{\begin{array}{l}
F_{p}=-\alpha V \\
I=\alpha \dot{u}-C \dot{V}
\end{array}\right.
$$

式中, $C$ 为压电元件的受夹电容; $\alpha$ 为力因子; $I$ 为 流经压电元件的电流。

由式(1)两边同乘速度, 并对时间积分, 可得能 量表达式(3)

$$
\begin{gathered}
\int F u \mathrm{~d} t=\frac{1}{2} M \dot{u}^{2}+\frac{1}{2} K_{E} u^{2}+ \\
\int C_{s} \dot{u}^{2} \mathrm{~d} t+\int \alpha V \dot{u} \mathrm{~d} t
\end{gathered}
$$

输入的总能量 $\int F u \mathrm{~d} t$ 包括: 动能 $M \dot{u}^{2} / 2$ 、弹性 势能 $K_{E} u^{2} K_{E} u^{2} / 2$ 、机械耗能 $\int C_{s} \dot{u}^{2} \mathrm{~d} t$ 和机电转换能 $\int \alpha V \dot{u} \mathrm{~d} t$ 。

半主动振动控制的原理就是根据能量守恒定 理, 在输入能量不变的前提下, 提高机电转换能来 
减小机械振动的能量, 从而达到振动控制的目的。

\section{2 电感同步开关阻尼技术 SSDI}

电感同步开关阻尼技术 SSDI 是在压电元件的两 极串联电感和开关, 构成振荡控制电路, 如图 2 所示, 当结构振动的位移达到极值时闭合开关, 此时电路发 生振荡。当压电元件的电压实现翻转时, 也即共振半 个周期, 迅速断开开关。通过控制开关的闭合与断开, 使得压电元件上的电压所产生的控制力始终与结构 运动的速度方向相反, 即产生的作动力始终阻碍结构 的振动, 从而达到了振动控制的目的。

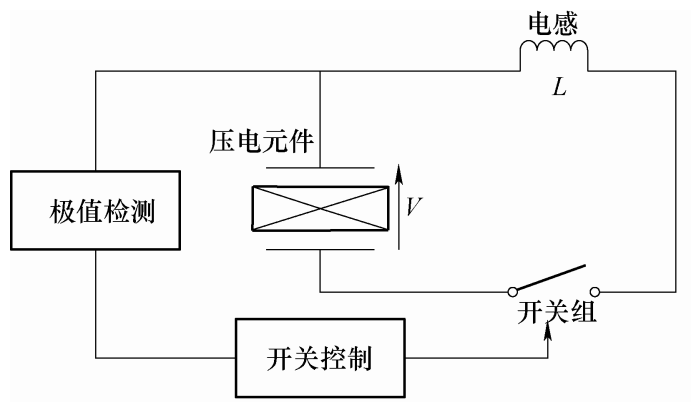

(a) 控制原理图

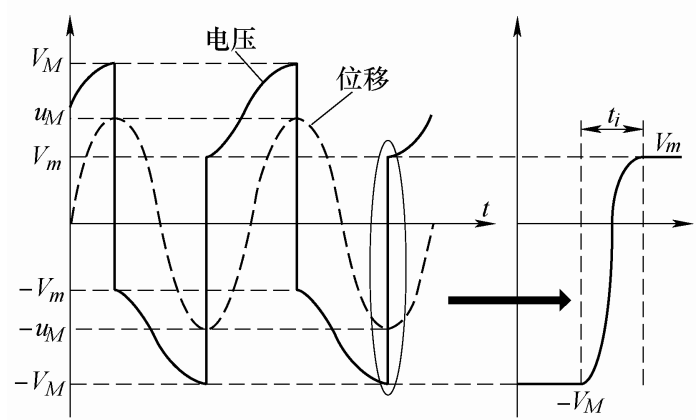

(b) 控制电压与位移关系

图 2 SSDI 控制方法

\section{3 基于外加电压源的同步开关阻尼技术 SSDV}

为了提高控制效果, 可在开关电路中插入电压 源, 即 SSDV 方法, 提高压电器件两端的电压, 从 而产生更大的有效控制力。

如图 3 所示, 在压电元件回路中串联一个极性 相反的电压源组, 以此来补充由于开关网络消耗压 电电容存储的那部分能量。由于外加电压源 $V_{c c}$ 的 作用, 增加了压电元件上的电压, 从而增强了控制 阻尼效果。

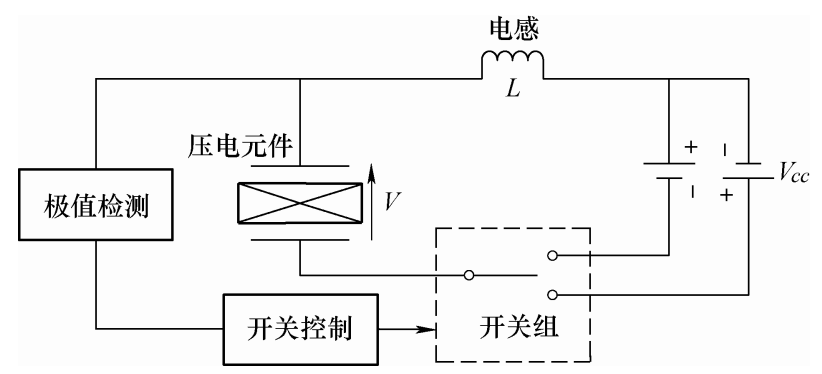

图 $3 \mathrm{SSDV}$ 控制原理图
SSDV 位移和电压曲线如图 4 所示, 当压电元 件的位移达到最大值时，使开关闭合，此时电路中 的 LC 振荡器将发生谐振, 当此谐振运动经历半个 周期 $t_{i}$ 时，迅速断开开关，压电元件上的电压将发 生翻转, 在压电元件上的电压达到正极大值时, 开 关使压电元件上的电压翻转后再外加一个电源 $-V_{c c}$, 压电元件上的电压达到负极大值时, 开关使 压电元件上的电压翻转后再附加一个 $+V_{c c}$ 。

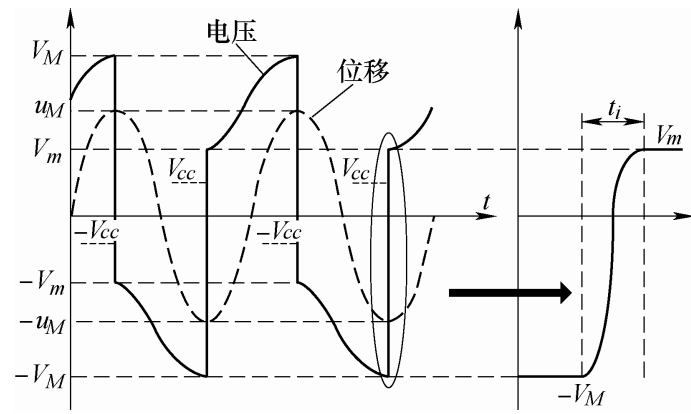

图 $4 \mathrm{SSDV}$ 控制电压与位移关系

SSDV 一个周期内机电转换能

$$
E_{t}=\int \alpha V \mathrm{~d} u=\left(\frac{4 \alpha^{2}}{C_{0}} u_{M}^{2}+4 \alpha u_{M} V_{c c}\right) \frac{1+\gamma}{1-\gamma}
$$

\section{4 自适应 SSDV 控制}

在传统的 SSDV 控制中电压源 $V_{c c}$ 的值通常是 恒定的, 电压源 $V_{c c}$ 过小则无法满足系统控制性能 要求, 过大则可能使控制力过大而反激起结构振动。 为了解决传统 SSDV 电压源大小选择的问题, LEFEUVRE 等 ${ }^{[13]}$ 提出了改进的 SSDV 方法, 电路中 外加电压源 $V_{c c}$ 的值不是固定的, 而是随着结构振 动大小自适应改变。当结构振动幅度较大, $V_{c c}$ 的值 能够自适应的变大，提高控制效果，当结构振动幅 度较小, $V_{c c}$ 能够跟着变小, 提高系统的鲁棒性。其 变化率为

$$
V_{c c}=\beta u_{\mathrm{ext}}
$$

$V_{c c}$ 在位移极值处更新, 且其始终与位移极值成 正比, 其比值 为一恒定正值。采用改进的 SSDV 技术从理论上可以避免经典 SSDV 的缺点, 避免在 瞬态激励下的控制失稳问题。

\section{2 仿真模型建立与仿真模拟}

在 ABAQUS 中根据真实展开臂试验系统建立 带压电元件的展开臂仿真模型，如图 5 所示。展开 臂一端固支, 一端自由, 总长度 $15 \mathrm{~m}$, 自由端加载 $200 \mathrm{~kg}$ 负载。压电元件布设在展开臂杆结构表面。 展开臂自由端部的负载和臂杆上的零重力气足用集 
中质量模拟, 臂杆之间的关节以弹簧等效(图 5)。调 节各个臂杆的刚度, 得到和真实展开臂系统模态测 试结果相吻合的前两阶固有振动频率, 如图 6 所示, 频率分别为 $0.085 \mathrm{~Hz}$ 和 $1.125 \mathrm{~Hz}$ 。调节系统的阻尼 系数, 使其一阶阻尼比为 $0.6 \%$ 。

图 5 带压电元件的展开臂仿真模型
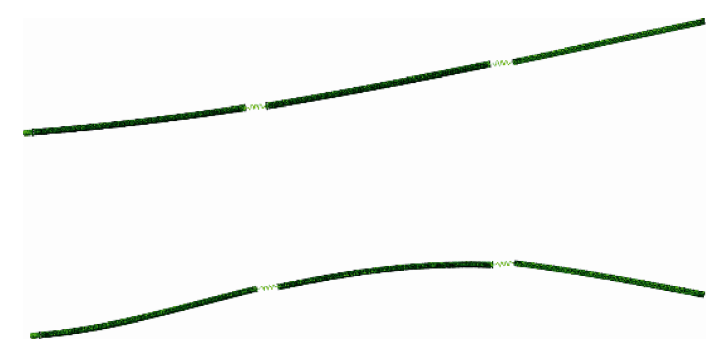

图 6 展开臂前两阶模态

\section{1 瞬态载荷激励下的未加控制展开臂仿真}

模拟瞬态载荷作用, 在展开臂端部施加 $100 \mathrm{~mm}$ 初始位移, 然后释放, 自由衰减情况下展开臂端部 位移曲线如图 7 所示。

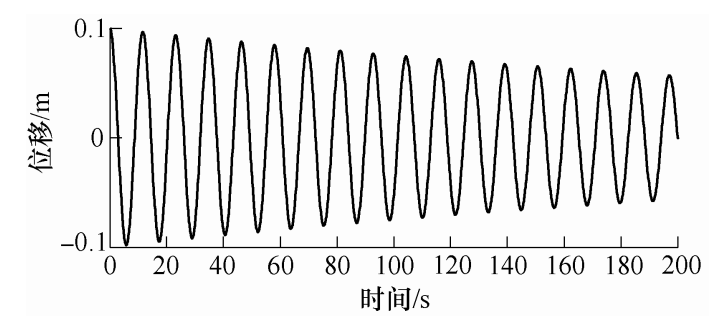

图 7 展开臂端部自由衰减位移曲线(初始位移 $100 \mathrm{~mm}$ )

\section{2 瞬态载荷激励下展开臂 SSDV 控制仿真}

瞬态载荷作用下, 对初始位移 $100 \mathrm{~mm}$ 工况, 通过展开臂结构表面的压电元件进行控制, 展开臂 端部位移曲线和压电元件两端电压曲线仿真结果如 图 8 所示。

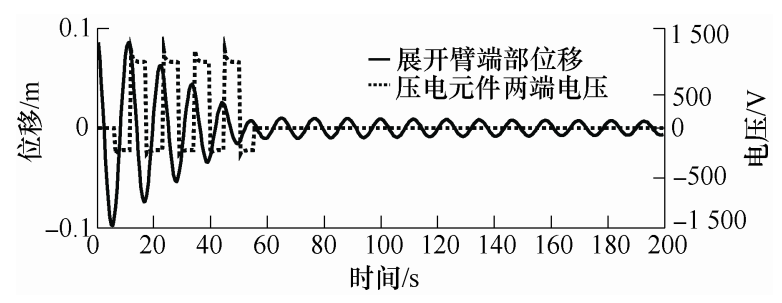

图 8 展开臂端部位移曲线和压电元件电压 曲线(初始位移 $100 \mathrm{~mm}$ )

展开臂系统等效阻尼比提高至 $8 \%$, 压电元件 最高控制电压达 $1166 \mathrm{~V}$, 反向最高电压-388 V。最 终系统振动控制在 $10 \mathrm{~mm}$ 的范围内。

在上述 SSDV 控制中, 控制电压始终保持确定 值, 而在将展开臂的位移控制到小位移幅值时, 容 易引起低位移高电压下的控制失稳。
图 9 为瞬态载荷下, 控制电压过大, 反激起结 构振动, 进而导致控制系统开关切换混乱, 系统在 一定范围内振荡。更严重的情况甚至可能导致最终 控制发散(图 10)。

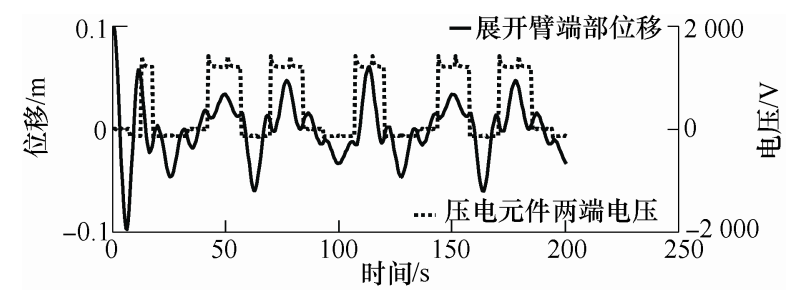

图 9 控制失稳时展开臂端部位移曲线和压电元件电压曲线

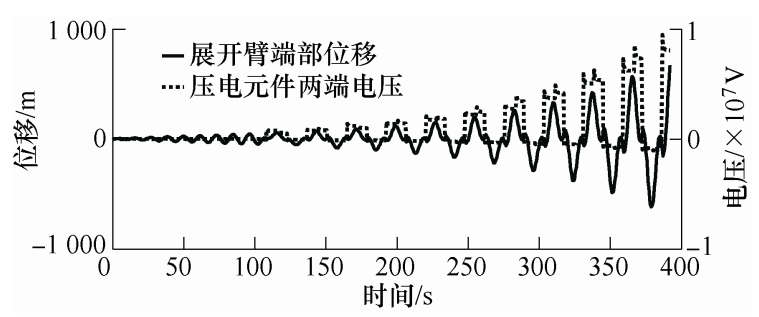

图 10 控制失稳时自激振荡曲线

\section{3 瞬态载荷激励下展开臂自适应 SSDV 控制仿真}

因此在控制时需要实时地根据端部位移自适应 地调整控制电压的大小，即在控制中加入反馈环节 以调整电压的大小, 避免结构的控制失稳。

对于端部初始位移 $100 \mathrm{~mm}$ 的瞬态载荷工况, 采用与端部位移成正比的自适应控制电压进行控 制, 仿真结果如图 11 所示。展开臂系统等效的阻尼 比为 $5.4 \%$, 虽然与 SSDV 控制时相比有所降低, 但 避免了在低位移时位移与控制力不匹配的控制失稳 问题。此外, 在低位移幅值的情况下由于避免了结 构失稳, 控制系统仍然能够对结构进行持续的控制, 控制的精度大大提高, 最终系统收玫在 $3 \mathrm{~mm}$ 的小 幅振动中。

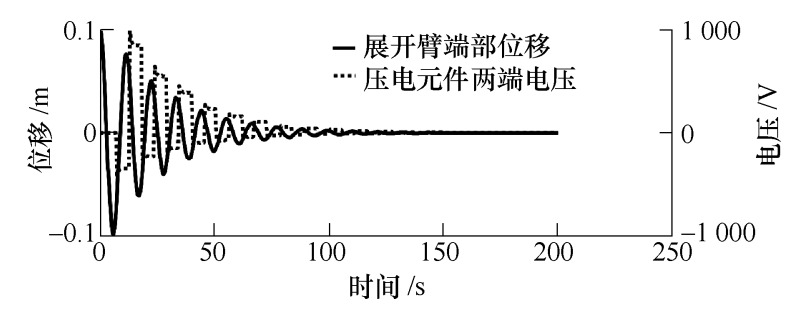

图 11 自适应控制仿真展开臂端部位移曲线和 压电元件电压曲线(初始位移 $100 \mathrm{~mm}$ )

\section{3 试验验证}

针对柔性天线展开臂进行振动抑制试验, 如图 12、13 所示。搭建基于同步开关阻尼技术的半主动控 制试验平台, 展开臂结构总长度 $15 \mathrm{~m}$, 一端固支, 一 端自由, 并且在自由端加载 $200 \mathrm{~kg}$ 重物模拟天线负 
载。利用多个气足完成零重力模拟。针对需要控制的 展开臂一阶弯曲模态进行传感器和驱动器的布设。

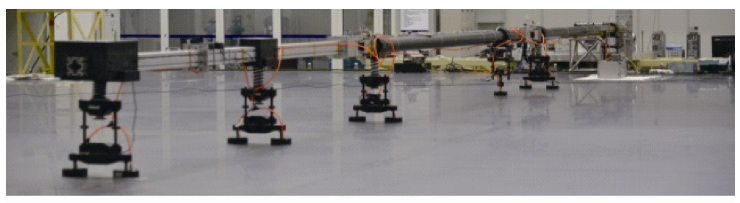

图 12 柔性天线展开臂

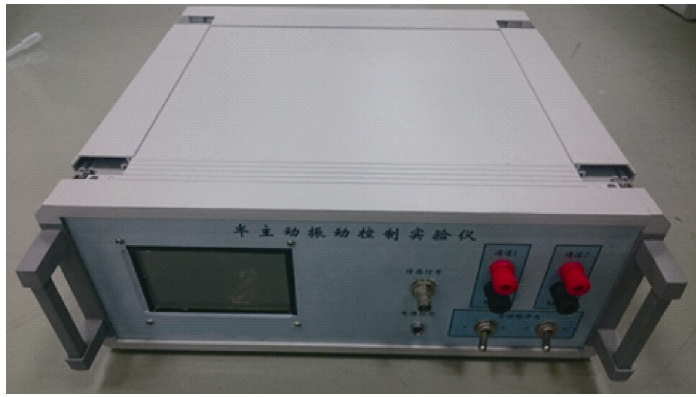

图 13 半主动控制器

试验中位移信号由设置在展开臂结构前端和根 部的激光位移测振仪进行采集, 结构应变通过布置 在根部的压电传感器获得。试验中压电驱动由布置 在展开臂结构根部的多片并联的压电单元提供。控 制系统通过采集得到的根部压电传感元件的电信 号, 经半主动控制器产生开关控制信号, 传输给压 电驱动器执行。半主动控制器共有两个通道, 为避 免驱动器在开路状态下压降过大, 在其两端并联有 电容。

在结构端部施加 $100 \mathrm{~mm}$ 初始位移, 然后释放, 自由衰减情况下结构端部位移曲线如图 14 所示。系 统的等效阻尼比为 $0.6 \%$ 。

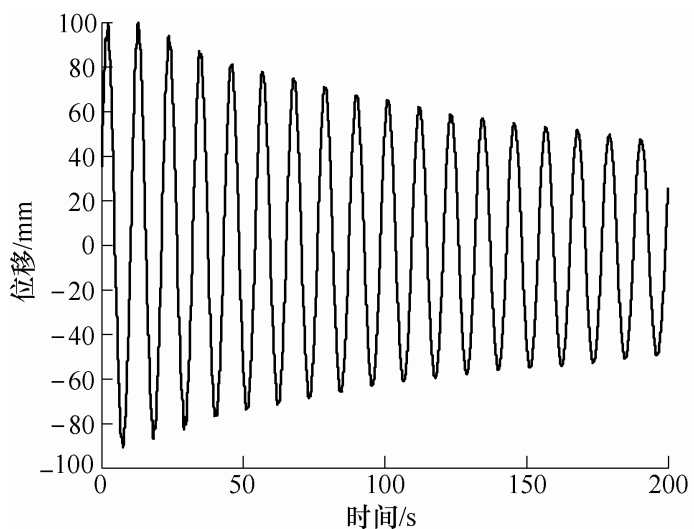

图 14 展开臂自由衰减端部位移曲线(初始位移 100mm)

试验从瞬态载荷激励与稳态载荷激励两方面对 控制系统进行验证：瞬态载荷激励给予臂杆端部一 个 $100 \mathrm{~mm}$ 初始位移, 通过控制加速系统稳定收玫, 提高系统的等效阻尼比; 稳态载荷激励在臂杆根部 通过激振器产生 $50 \mathrm{~mm}$ 的稳态振动, 通过控制系统 产生等效的附加阻尼力减小其稳态振动幅值。

\section{1 瞬态载荷激励下 SSDV 振动抑制试验}

对于初始位移 $100 \mathrm{~mm}$ 的瞬态载荷激励, 施加 SSDV 控制前后结构端部位移和电压曲线如图 15、 16 所示, 电压源电压 $V_{c c}$ 为 $130 \mathrm{~V}$ 。施加控制后的等 效阻尼比约为 $5.83 \%$, 系统阻尼比提高了 $5.2 \%$ 。采 用约 $100 \mathrm{~s}$ 将系统位移从 $100 \mathrm{~mm}$ 控制到 $5 \mathrm{~mm}$ 范围 内。而依靠系统自身阻尼 $100 \mathrm{~s}$ 仅能衰减至 $60 \mathrm{~mm}$ 。 最高控制电压达 $1167 \mathrm{~V}$, 反向最高电压 $-329 \mathrm{~V}$ 。

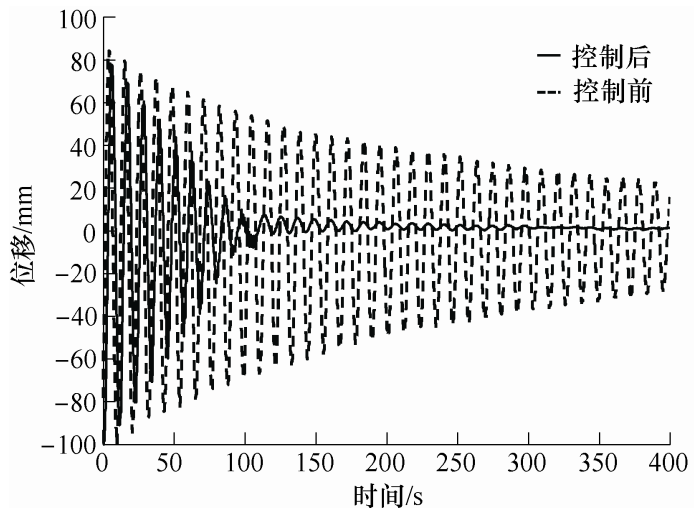

图 15 控制前后展开臂端部位移曲线(初始位移 $100 \mathrm{~mm}$ )

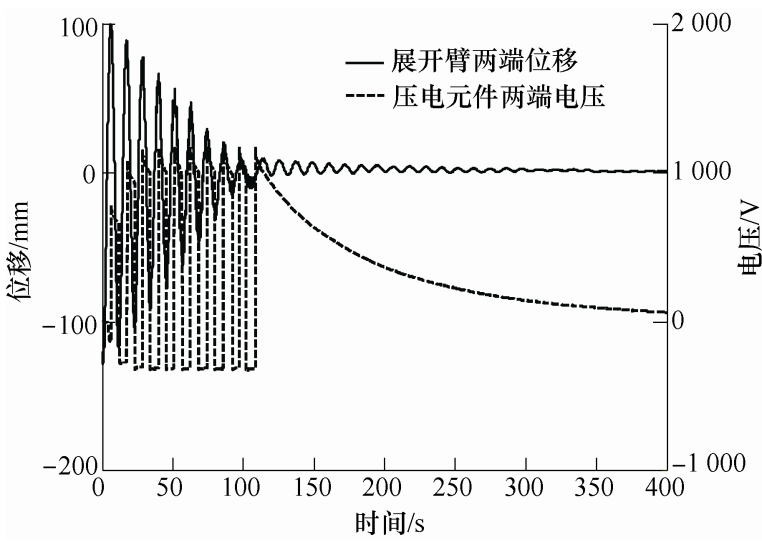

图 16 展开臂端部位移曲线和压电元件 电压曲线(初始位移 $100 \mathrm{~mm}$ )

\section{2 瞬态载荷激励下自适应 SSDV 振动抑制试验}

对于初始位移 $100 \mathrm{~mm}$ 的瞬态载荷激励, 施加 自适应控制后结构端部位移和电压曲线如图 17 所

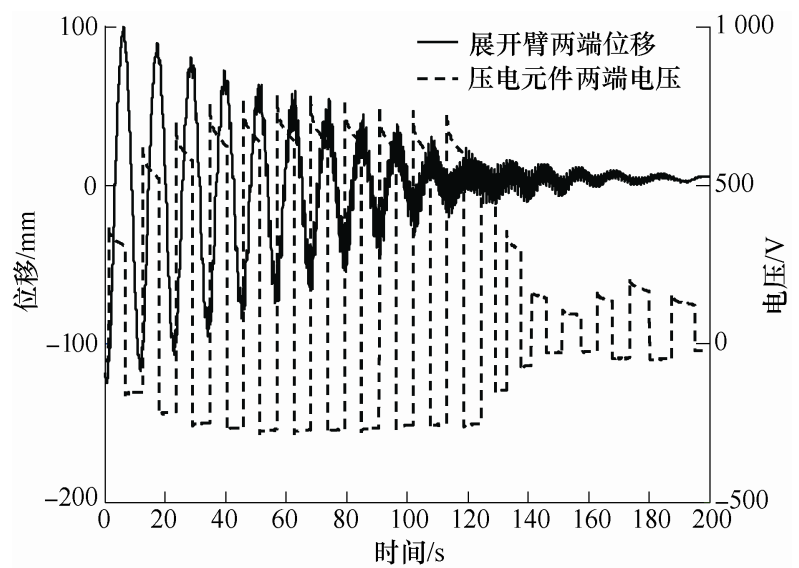

图 17 自适应控制后展开臂端部位移曲线和压电元件电压 曲线(初始位移 $100 \mathrm{~mm}$ ) 
示, 控制电压随着系统位移的减小而减小, 始终对 结构进行控制而未激起结构振动。最终的控制精度 较高, 达到了约 $2 \mathrm{~mm}$ 的控制精度。施加控制后的 等效阻尼比约为 $4.03 \%$, 最高控制电压达 $777 \mathrm{~V}$, 反 向最高电压 $-282 \mathrm{~V}$ 。

\section{3 稳态载荷振动抑制试验}

对天线展开臂根部施加一个稳态激励, 使其端 部产生 $50 \mathrm{~mm}$ 振幅, 得到控制前后结构端部位移曲 线如图 18 所示。控制前结构稳态振动幅值为 50 $\mathrm{mm}$, 控制后结构端部振动幅值降低为 $10 \mathrm{~mm}$ 。对 采集得到的位移信号做快速傅里叶分析, 得到控制 前后频谱图如图 19 所示。控制前基频 $0.09 \mathrm{~Hz}$ 处幅 值为 $25.82 \mathrm{~dB}$, 控制后降低为 $8.977 \mathrm{~dB}$, 幅值降低 了 $16.843 \mathrm{~dB}$ 。

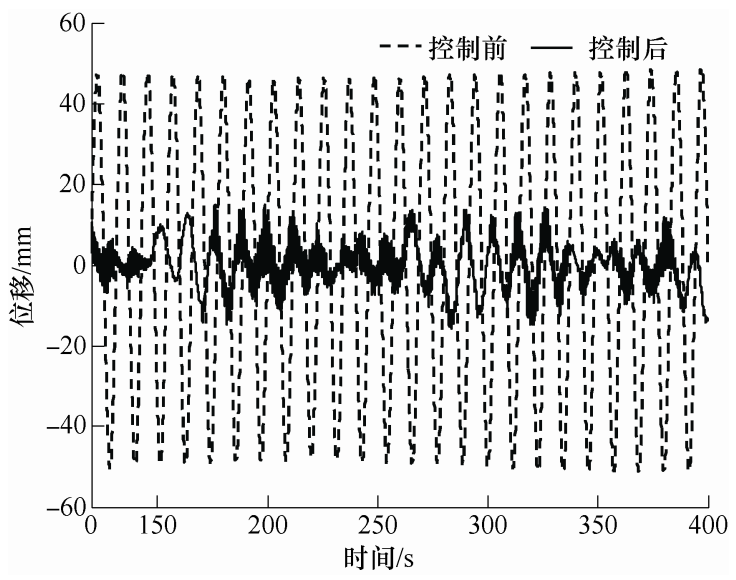

图 18 稳态激励下(幅值 $50 \mathrm{~mm}$ ) 结构控制前后端部位移曲线

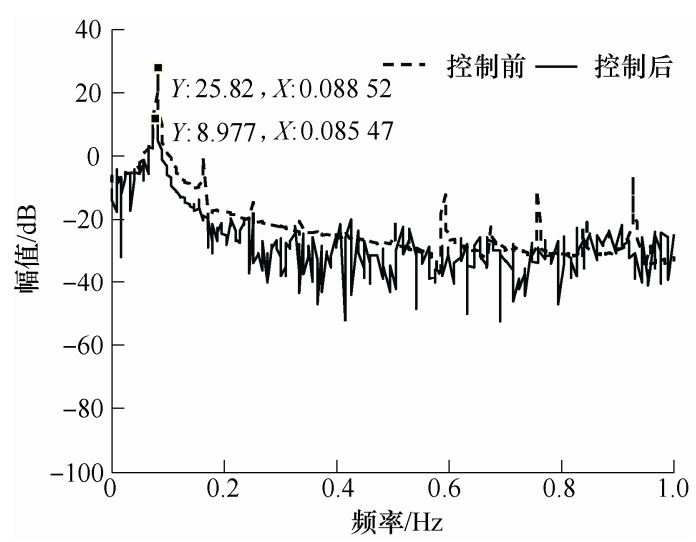

图 19 稳态幅值 $50 \mathrm{~mm}$ 时控制前后幅频特性曲线

\section{4 结论}

本文针对大型柔性展开臂振动抑制开展了研 究, 提出了采用自适应半主动控制方法对柔性展开 臂进行振动抑制。试验结果表明自适应半主动振动 控制能够有效地增大系统等效阻尼, 加速系统自由 衰减, 减小系统稳态响应振动的幅值。与普通半主 动控制相比, 能提高控制精度, 降低控制电压, 同
时解决了低位移高电压容易引起的控制失稳问题, 更适合工程应用。半主动振动控制相比其他控制方 法具有不需要精确的结构振动模型, 控制效果较稳 定的优点, 能够在柔性展开臂振动控制中得以应用。

\section{参 考 文 献}

[1] GUYOMAR D, BADEL A. Nonlinear semi-passive multimodal vibration damping: An efficient probabilistic approach[J]. Journal of Sound and Vibration, 2006, 294(1-2): 249-268.

[2] RICHARD C, GUYOMAR D, AUDIGIER D, et al. Semi-passive damping using continuous switching of a piezoelectric device[C]// In Proceedings of the SPIE Smart Structures and Materials Conference: Passive Damping and Isolation, 1998, San Diego, USA, 1998: 3672: 104-111.

[3] RICHARD C, GUYOMAR D, AUDIGIER D, et al. Semi-passive damping using continuous switching of a piezoelectric device[J]. Proceedings of the SPIE International Symposium on Smart Structures and Materials: Passive Damping and Isolation, 1999, 3672: 104-111.

[4] RICHARD C, GUYOMAR D, AUDIGIER D, et al. Enhanced semi-passive damping using continuous switching of a piezoelectric device on an inductor[J]. Proceedings of the SPIE International Symposium on Smart Structures and Materials: Damping and Isolation, 2000, 3989: 288-299.

[5] LEFEUVRE E, GUYOMAR D,PETIT L, et al. Semi-passive structural damping by synchronized switching on voltage sources[J]. Journal of Intelligent Material Systems and Structures, 2006, 17(8-9): 653-660.

[6] GRASSO E, TOTARO N, JANOCHA $\mathrm{H}$, et al. Piezoelectric self-sensing actuators for high voltage excitation[J]. Smart Materials and Structures, 2013, 22(6): 065018.

[7] BAO B, TANG W. Semi-active vibration control featuring a self-sensing SSDV approach[J]. Measurement, 2017, 104: 192-203.

[8] HUANG W, WANG X, ZHANG J, et al. Some advances in the vibration control of aerospace flexible structures[J]. Advances in Mechanics, 1997, 27(1): 6-19.

[9] MAKIHARA K, ONODA J, MINESUGI K. Novel approach to self-sensing actuation for semi-active vibration suppression[J]. AIAA Journal, 2006, 44(7): 1445-1453.

[10] MAKIHARA $\mathrm{K}$, ONODA J , MINESUGI K. Low-energy-consumption hybrid vibration suppression based on an energy-recycling approach[J]. AIAA Journal, 2005, 43: 1706-1715. 
[11] MAKIHARA K，ONODA J，MINESUGI K. Behavior of piezoelectric transducer on energy-recycling semi-active vibration suppression[J]. AIAA Journal, 2006, 44 : 411-413.

[12] MAKIHARA K, ONODA J. Investigation of performance in suppressing various vibrations with energy-recycling semi-active method[J]. Acta Astronautica, 2006, 58(10): 506-514.

[13] LEFEUVRE E, BADEL A, PETIT L, et al. Semi-passive piezoelectric structural damping by synchronized switching on voltage sources[J]. Journal of Intelligent Material Systems and Structures, 2006, 17: 653-660.
[14] SABATINI M, GASBARRI P, MONTI R, et al. Vibration control of a flexible space manipulator during on orbit operations[J]. Acta Astronautica, 2012, 73(2): 109-121.

[15] GARRIDO H, CURADELLI O, AMBROSINI D. Semi-active friction tendons for vibration control of space structures[J]. Journal of Sound and Vibration, 2014, 333(22): 5657-5679.

作者简介: 王晓宇(通信作者), 女, 1981 年出生, 博士, 高级工程师, 硕士研究生导师。主要研究方向为航天器结构设计与分析, 动力学与振 动控制。

E-mail: antlb@163.com 